\title{
Quantifying the Effects of Acquisition Parameters in Cardiac SPECT Imaging and Comparison with Visual Observers
}

\author{
Johannes Zeint1*, Student Member IEEE, A. Hans Vija*, James T. Chapman, Eric G. Hawman, Joachim Hornegger, \\ Members, IEEE
}

\begin{abstract}
Significant debate remains whether the established imaging protocols for myocardial perfusion SPECT delivers optimal image quality. This debate has been intensified with the introduction of advanced iterative reconstruction techniques which differ considerably from the FBP reconstruction technology commonly available when the current protocols were established. In order to characterize and classify image quality affecting factors, simulations combined with time consuming observer studies are often used.

In this work we seek to simplify these image assessment tasks by performing numerical characterizations based on real data, and try to unite quantitative performance with visual impression derived from human observer studies.

For this purpose, we assess the response of cardiac image quality to variations in acquisition and reconstruction protocol. We analyze anthropomorphic phantom data derived from large dimensioned acquisitions via subsampling in terms of counts and spatial and angular resolution. Data is reconstructed using FBP and OSEM-3D ("Flash3D") with parameter variations and the reconstructed volumes are quantitatively analyzed for cardiac relevant features like myocardial uniformity and resolution. Receiver Operating Characteristic (ROC) studies are performed for selected imaging parameters. Results obtained characterize the sensitivity of the cardiac image quality to some changes in the imaging protocol like angular step, orbit radius and collimation and show correlations of key quantitative measures and related ROC behavior.
\end{abstract}

\section{INTRODUCTION}

$\mathrm{I}_{\mathrm{N}}^{\mathrm{N}}$ recent years single photon emission tomography systems $\mathbf{I}_{\text {gained in image quality performance from advances }}$ throughout the entire image formation chain. These improvements also result in increasing options and variability for the imaging protocols of clinical workflows.

In the field of myocardial perfusion SPECT this leads to the question whether the current established imaging methodologies $[1,2]$ still deliver the optimal image quality.

To assess how different acquisition and reconstruction parameters might affect the image quality in SPECT images

Manuscript received November 17, 2006. Asterisk indicates corresponding author.

*Johannes Zeintl is a student with the University of Erlangen-Nürnberg, Institute of Pattern Recognition, Erlangen, Germany. He is currently with Siemens Molecular Imaging (e-mail: Johannes.Zeintl.ext@siemens.com).

*A. Hans Vija (e-mail: hans.vija@siemens.com), James T. Chapman, Eric G. Hawman are with Siemens Medical Solution USA, Inc., Molecular Imaging, Hoffman Estates, IL 60195, USA.

Joachim Hornegger is with University of Erlangen-Nürnberg, Institute of Pattern Recognition, Erlangen, Germany. simulations and mathematical models are often used [9-12]. These procedures indeed have high potential and deliver significant and satisfying results however inevitable uncertainties in system modeling are introduced.

Simulations are often combined with task-based observer studies to validate the results in terms of visually detectable features. Receiver-operating-characteristic (ROC) studies are a powerful means to assess changes in image quality of clinical relevance however they are laborious and time-consuming. To some extent, the data sets used for these studies often require considerable fine tuning to deliver meaningful results, which may not always reflect the clinical reality. This leads to the question whether ROC analysis for cardiac SPECT may in some instances be represented by certain quantitative measures.

In this work we quantify the effects of acquisition parameters by investigating cardiac image data, acquired with a cardiac torso phantom and a variety of different system setups. Two different reconstruction methods, FBP and OSEM-3D ("Flash3D") are applied and the cardiac volume is analyzed in terms of cardiac relevant metrics like wall thickness and uniformity in the myocardium.

Furthermore, exclusive imaging parameters were selected to be investigated in human ROC studies. Results from both the quantitative analyses and the observer studies were compared to show correlations. For cardiac imaging this may indicate the behavior of ROC results based on quantitative measurements only.

\section{MATERIALS AND MethodS}

\section{A. Imaging Instrumentation}

The imaging system used for data acquisition was a dualheaded SPECT-CT hybrid camera (Symbia ${ }^{\circledR}-\mathrm{T} 6$, Siemens Medical Solutions). Three different low energy collimator types were used for comparison: Low Energy All Purpose (LEAP), Low Energy High Resolution (LEHR), and Low Energy Ultra High Resolution (LEUHR). The sensitivity ratio of these collimators is: LEAP:LEHR:LEUHR $=1.5: 1: 0.5$ and the geometric resolution at $10 \mathrm{~cm}$ is $8.3 \mathrm{~mm}, 6.4 \mathrm{~mm}$, and $4.6 \mathrm{~mm}$, respectively. 


\section{A. Phantom Setup}

The phantom used for acquisition was an anthropomorphic torso phantom (Data Spectrum, Hillsborough, NC), an acrylic glass cylinder with cardiac, liver, lung, and spine components (see Fig.1). The cardiac insert consists of two chambers, simulating the left ventricular bloodpool and the myocardial wall with a true wall thickness of $10 \mathrm{~mm}$. The phantom was loaded with ${ }^{99}$ Technetium adjusting the activity concentration ratio according to Table I. For abnormal studies a fillable lesion with an angular extent of $45^{\circ}$ and a length of $20 \mathrm{~mm}$ (displacement volume: $4.7 \mathrm{~mL}$ ) was mounted in the mid-inferior region of the cardiac insert and filled the entire radial extension of the wall. The activity concentration in the defect was $12.5 \%$ below the surrounding myocardium (see Table I).

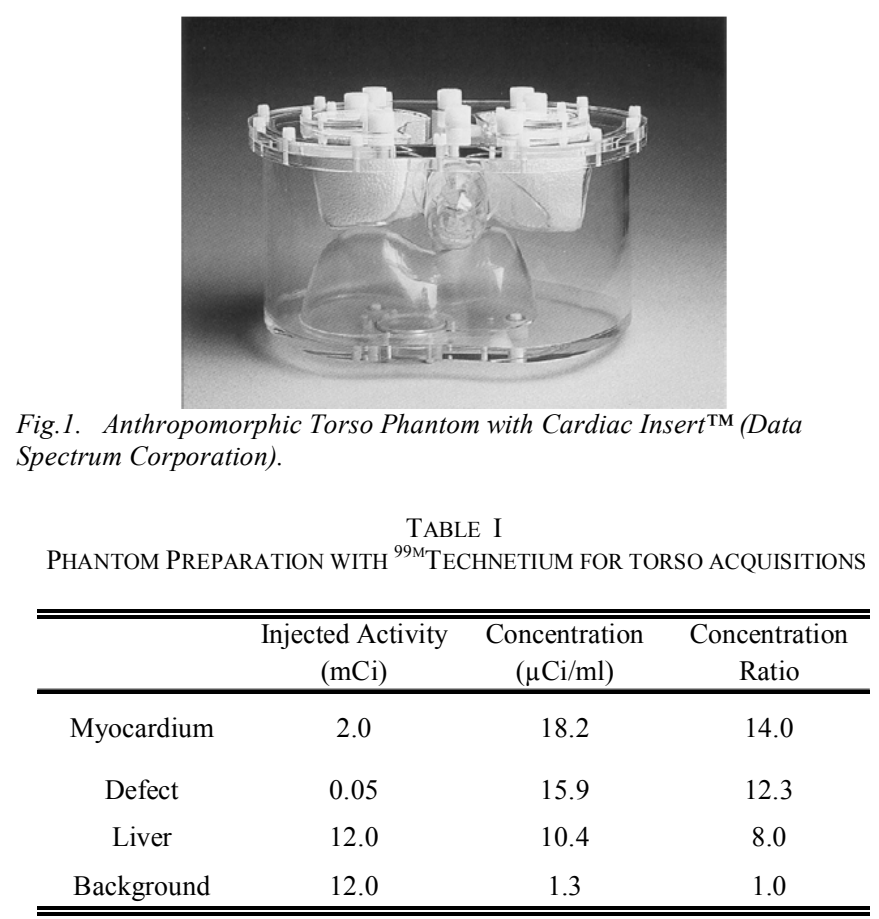

\section{B. Data Acquisition}

The image data was acquired using the three collimators mentioned above. The cardiac insert was acquired both in air, in order to investigate basic principles without image degradation caused by attenuation, as well as within the torso casing. Table II provides an overview of the acquisition parameter used for both phantom setups. The insert in air was acquired by varying the radius of a circular $360^{\circ}$-orbit. The scan orbit range of the torso extended from $45^{\circ}$ left posterior oblique to $45^{\circ}$ right anterior oblique where the detectors followed the human body-like shape of the phantom in a noncircular orbit to minimize the distance to the object. In this range high-count projections in one-degree angular steps were acquired with a 256x256 matrix size and zoom 1.45 (see Table II). These data sets served as high level starting point for further processing.
TABLE II

KEY ACQUISITION PARAMETERS FOR THE CARDIAC INSERT ${ }^{\mathrm{TM}}$ IN AIR AND WITHIN THE TORSO CASING.

\begin{tabular}{lcc}
\hline \hline & Insert & Torso \\
\hline Head Separation Angle & $180^{\circ}$ & $90^{\circ}$ \\
Scan Range & $360^{\circ}$ & $180^{\circ}$ \\
Angular Step & $3^{\circ}$ & $1^{\circ}$ \\
Orbit & circular & non-circular \\
Radius (cm) & $15,20,25,30$ & N/A \\
Pixel Size (mm) & 2.4 & 1.65 \\
Matrix Size & $128 \times 128$ & $256 \times 256$ \\
Total Counts & $6000 \mathrm{k}$ & $90000 \mathrm{k}$ \\
Counts/view & $100 \mathrm{k}$ & $1000 \mathrm{k}$ \\
Count Density in & $24-27$ & $44-52$ \\
myocardium $\left(\mathrm{cts} / \mathrm{mm}^{2}\right)$ & & \\
\hline \hline
\end{tabular}

Note: Count density is given for LEHR collimation.

\section{Projection Data Processing and Reconstruction}

The projection data of the cardiac insert in air was not further processed and reconstructed with both Filtered Backprojection (Butterworth filter of order 5) and OSEM 3D with collimator and detector response compensation ("Flash3D"). Table IV provides detailed information about the reconstruction parameters used.

For further processing of the torso data a tool was developed which allows DICOM compatible handling and manipulation of projection data sets. Applying it to the acquired high-count projection images of the torso this data was subsampled in various respects:

Count reduction to clinical levels and below was done by binomial subsampling. By definition the binomial distribution gives the discrete probability distribution of obtaining exactly $n$ successes out of $N$ Bernoulli trials where the result of each Bernoulli trial is true with probability $p$. According to this, for each pixel value $N$ a new count $n$ was obtained using the subsampling fraction $p$. Applied to a Poisson deviate (a count in a pixel) the outcome is a new Poisson deviate whose mean is reduced to a fraction $p$. Thus, a new subsampled Poisson distribution is generated. However, multiple realizations of the same initial Poisson deviate are not statistical independent and their variance is $N p(1-p)$ instead of $N p$, yet with $N \rightarrow \infty$ and $p \rightarrow 0$ the error is minimized.

Accordingly various sets of quasi-independent low count deviates were created from the high count data sets using a $p$ of approximately 0.01 .

In order to vary angular separation of the projections, the desired frames were extracted from the one-degree data set and recombined to a valid projection set. Note that there was no frame interpolation or summing applied to the projections. 
Matrix size was changed by rebinning of the pixel grid and summing the corresponding values of the merged pixels.

Table III gives an overview how the projection data (only LEHR data was used) was processed to get the different count levels and angular separations. Thereby the matrix size was set to $64 \times 64$. The data for the human observer studies were generated by count-reduction to levels of gated time bins. This level was chosen in order to maintain AUC values in a range that provide good statistical power and also to offer clinical relevance. Table III shows the reconstruction parameter used for creating the data sets for the ROC studies. Note that both the number of updates (iterations*subsets) and the number of views per subset maintained the same throughout the three different angular steps. The full-width-at-half-maximum (FWHM) of the Gaussian post-smoothing subsequent to the iterative reconstruction was set to two times the pixel size.

TABLE III

COUNT LEVELS AND OSEM 3D PARAMETERS FOR THE ROC DATA SETS

\begin{tabular}{|c|c|c|c|c|c|c|}
\hline $\begin{array}{c}\text { Total } \\
\text { Counts }\end{array}$ & $\begin{array}{c}\text { Counts/ } \\
\text { view }\end{array}$ & $\begin{array}{l}\text { Count density } \\
\text { in } \\
\text { myocardium } \\
\left(\text { cts } / \mathrm{mm}^{2}\right)\end{array}$ & $\begin{array}{c}\text { Angular } \\
\text { step } \\
\text { (degrees) }\end{array}$ & Iterations & Subsets & $\begin{array}{c}\text { \# OSEM } \\
\text { Updates }\end{array}$ \\
\hline \multirow{3}{*}{$450 \mathrm{k}$} & $7.5 \mathrm{k}$ & $0.23-0.35$ & 3 & 6 & 6 & 36 \\
\hline & $15 \mathrm{k}$ & $0.45-0.70$ & 6 & 12 & 3 & 36 \\
\hline & $22.5 \mathrm{k}$ & $0.75-0.90$ & 9 & 18 & 2 & 36 \\
\hline \multirow{3}{*}{$450 \mathrm{k}$} & $7.5 \mathrm{k}$ & $0.23-0.35$ & 3 & 6 & 12 & 72 \\
\hline & $15 \mathrm{k}$ & $0.45-0.70$ & 6 & 12 & 6 & 72 \\
\hline & $22.5 \mathrm{k}$ & $0.75-0.90$ & 9 & 18 & 4 & 72 \\
\hline \multirow{3}{*}{$450 \mathrm{k}$} & $7.5 \mathrm{k}$ & $0.23-0.35$ & 3 & 12 & 12 & 144 \\
\hline & $15 \mathrm{k}$ & $0.45-0.70$ & 6 & 24 & 6 & 144 \\
\hline & $22.5 \mathrm{k}$ & $0.75-0.90$ & 9 & 36 & 4 & 144 \\
\hline
\end{tabular}

Note: The FWHM of the Gaussian post-smoothing was set to double the pixel size, respectively. Only LEHR collimation was tested in ROC studies.

\section{Quantitative Analysis}

For a quantitative analysis of the image data a new measurement tool, specific to the cardiac phantom, was developed with the aim to facilitate the characterization of the performance of an imaging system. It provides the user with a set of quality control images as well as quantitative measures.

The tool processes reconstructed reoriented and transversal cardiac SPECT data and calculates diagnostically important metrics like perfusion, wall FWHM, bloodpool and lesion contrast and attenuation performance. For a detailed description of all features incorporated see [13].

This work focused on two metrics: resolution, manifested in the wall FWHM, and differential nonuniformity of the cardiac wall. The definition of the differential nonuniformity is the standard deviation of the perfusion values in the basal and mid regions of the myocardium normalized to the mean value:

$$
\text { Nonuniformity }=\frac{\text { stddev }(\text { Base }, \text { Mid })}{\text { mean }(\text { Base }, \text { Mid })} \cdot 100 \%
$$

\section{E. Human Observer Study}

The observer study used a graphical user interface showing the user a series of short axis images with a $50 \%$ chance of a defect being present. Each observer was asked to rank the images in a 5 step discrete scale from definitely placebo to definitely lesion. Fig. 2 shows the truth model, a short axis slice extracted of the centroid of the defect (mid-inferior). The defect locations remained constant in the 6 'clock position. The images were displayed zoomed ( 6 times the original matrix size) using cubic interpolation and shown with a 256step color table (warm metal). The short axis slices were created by reorientation of the reconstructed volume and extraction of a single slice in the center of the lesion.

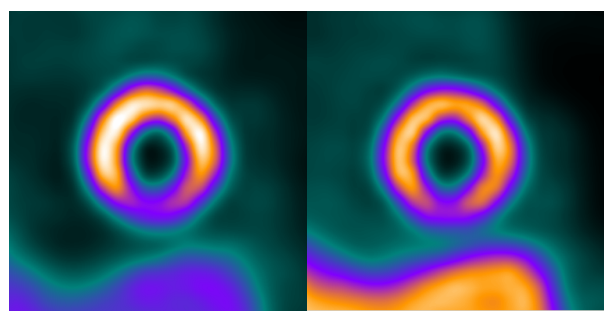

Fig.2. Short axis slice of the cardiac phantom showing the defect location with (right) and without attenuation correction (left). Slice is extracted of the defect centroid.

To assess each imaging parameter, the reader was presented with 100 images in random order, 50 with and 50 without defects. Prior to each ranking session the observer was able to run through training with feedback. In presence of a lesion a crosshair was displayed at the lesion location. The training could arbitrarily be ended by the user. A total of 12 parameters were tested (Table III) by 5 readers.

The ROC curves and the corresponding values for the area under the curve (AUC) and the standard error were calculated using the non-parametric model of Hanley, et al. [8]. Subsequently, the bootstrap method was applied to the ROC reader ratings (100 repeats per reader), in order to determine unbiased estimates and standard errors.

\section{RESULtS}

To give a visual impression for non-uniformity and wall FWHM, a comparison of reconstructed images (OSEM 3D and FBP) acquired with different collimators is shown in Fig.3. Note that the counts of the projection data for each collimator were set such that the same acquisition time can be assumed (LEAP: 245kcts/view; LEHR: 150kcts/view; LEUHR: $75 \mathrm{kcts} / \mathrm{view})$. For each collimator identical reconstruction parameters were used for FBP and Flash3D, respectively, in order to visualize principle effects of different collimation methods. Note that in general reconstruction parameters for Flash3D should be adjusted according to the 
counts available, in order to get an optimal tradeoff between resolution and uniformity.
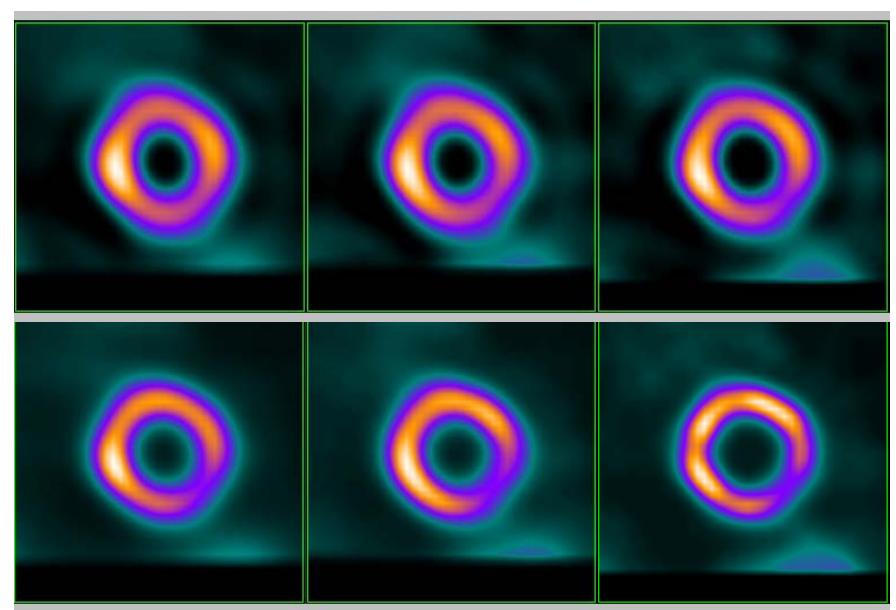

Fig.3. Example comparison of reconstructed images of the torso phantom acquired with three different collimators: LEAP (first column), LEHR (second column) and LEUHR (third column). Images were reconstructed with FBP (first row) and Flash3D (second row) using identical reconstruction parameters, respectively. Count levels were adapted towards constant acquisition time taking into account the collimator sensitivity.

TABLE IV

QUANTITATIVE RESULTS FOR THE RECONSTRUCTED VOLUMES SHOWN IN FIGURE 3

\begin{tabular}{ccccccc}
\hline \hline & \multicolumn{3}{c}{ Non-Uniformity (\%) } & \multicolumn{3}{c}{ Wall FWHM (mm) } \\
& LEAP & LEHR & LEUHR & LEAP & LEHR & LEUHR \\
\hline FBP & 8.6 & 8.3 & 8.5 & 21.3 & 20.6 & 18.7 \\
Flash3D & 9.0 & 8.9 & 9.4 & 18.9 & 17.7 & 15.1 \\
\hline \hline
\end{tabular}

Results obtained by analyzing the image data of the cardiac insert in air show principle impacts of variations in the selected imaging and reconstruction parameters. Table $\mathrm{V}$ gives an overview how different orbit radii, collimators, and reconstruction methods and parameters affect the myocardial wall FWHM and uniformity. The quantitative values shown are measured for one reconstructed volume, respectively. Analysis of multiple images reconstructed with the same parameters in general might show minor fluctuations depending on the count density however these single values are satisfactory to identify general trends as the signal-to-noise ratio was very high (high-count studies).

The values of the wall FWHM, which is a measure for both resolution and contrast, in Table V indicate that in Filtered Back-Projection no significant improvement can be achieved beyond a cut-off value of 0.4 . The same is true beyond 100 updates of OSEM-3D.

With respect to uniformity a slightly better performance can be observed with FBP in comparison to OSEM 3D. However, considering the FWHM, one can clearly see the benefits in iterative reconstruction. FBP not only results as a whole in poorer resolution, but there are also more significant differences between the collimation methods.
TABLE V

QUANTITATIVE RESULTS BASED ON RECONSTRUCTED IMAGES OF THE CARDIAC INSERT $^{\text {TM }}$ IN AIR

\begin{tabular}{|c|c|c|c|c|c|c|c|c|}
\hline & & & \multicolumn{3}{|c|}{ Non-Uniformity (\%) } & \multicolumn{3}{|c|}{$\begin{array}{c}\text { Avg. Wall FWHM } \\
(\mathrm{mm})\end{array}$} \\
\hline $\begin{array}{l}\text { Radius } \\
(\mathrm{cm})\end{array}$ & \multicolumn{2}{|c|}{ Reconstruction } & LEAP & LEHR & LEUHR & LEAP & LEHR & LEUHR \\
\hline \multirow{9}{*}{15} & \multirow{9}{*}{$\begin{array}{l}\text { OSEM 3D \# } \\
\text { updates }\end{array}$} & 10 & 6.0 & 5.0 & 5.7 & 16.2 & 14.5 & 13.0 \\
\hline & & 20 & 6.4 & 4.3 & 4.7 & 13.1 & 12.0 & 11.0 \\
\hline & & 40 & 5.5 & 3.6 & 4.1 & 11.2 & 10.5 & 9.9 \\
\hline & & 80 & 4.6 & 3.6 & 4.1 & 9.9 & 9.6 & 9.3 \\
\hline & & 100 & 4.7 & 3.7 & 4.2 & 9.5 & 9.2 & 9.0 \\
\hline & & 150 & 4.7 & 4.0 & 4.4 & 9.0 & 8.9 & 8.8 \\
\hline & & 200 & 4.7 & 4.3 & 4.6 & 8.7 & 8.8 & 8.8 \\
\hline & & 250 & 4.9 & 4.5 & 4.7 & 8.5 & 8.7 & 8.8 \\
\hline & & 300 & 5.0 & 4.7 & 4.8 & 8.4 & 8.6 & 8.8 \\
\hline \multirow{9}{*}{15} & \multirow{9}{*}{$\begin{array}{l}\text { FBP Filter } \\
\text { Cutoff }\end{array}$} & 0.1 & 10.0 & 10.2 & 10.1 & 29.9 & 30.7 & 31.5 \\
\hline & & 0.2 & 4.4 & 3.9 & 4.0 & 21.3 & 20.6 & 19.9 \\
\hline & & 0.2 & 4.0 & 3.2 & 3.5 & 18.4 & 17.5 & 16.8 \\
\hline & & 0.3 & 3.8 & 3.0 & 3.5 & 15.4 & 14.0 & 12.9 \\
\hline & & 0.4 & 4.0 & 3.3 & 4.0 & 14.8 & 13.1 & 11.9 \\
\hline & & 0.5 & 4.2 & 3.6 & 4.2 & 14.8 & 12.9 & 11.7 \\
\hline & & 0.6 & 4.3 & 3.8 & 4.3 & 14.7 & 12.9 & 11.7 \\
\hline & & 0.8 & 4.6 & 4.0 & 4.6 & 14.7 & 12.9 & 11.7 \\
\hline & & 1 & 4.7 & 4.2 & 4.7 & 14.7 & 12.9 & 11.6 \\
\hline \multirow{2}{*}{20} & \# updates & 150 & 6.3 & 4.2 & 4.0 & 9.3 & 9.1 & 8.6 \\
\hline & FBP cutoff & 0.4 & 4.3 & 3.4 & 3.4 & 17.3 & 14.4 & 12.5 \\
\hline \multirow{9}{*}{25} & \multirow{9}{*}{$\begin{array}{l}\text { OSEM 3D \# } \\
\text { updates }\end{array}$} & 10 & 7.2 & 6.1 & 5.2 & 22.4 & 18.0 & 14.7 \\
\hline & & 20 & 7.5 & 5.9 & 5.0 & 16.5 & 14.1 & 11.9 \\
\hline & & 40 & 7.5 & 5.4 & 4.1 & 13.3 & 11.8 & 10.3 \\
\hline & & 80 & 7.1 & 4.9 & 3.8 & 11.2 & 10.3 & 9.2 \\
\hline & & 100 & 7.2 & 4.9 & 3.7 & 10.6 & 9.9 & 9.0 \\
\hline & & 150 & 7.2 & 5.1 & 3.8 & 9.7 & 9.3 & 8.6 \\
\hline & & 200 & 7.3 & 5.2 & 4.0 & 9.3 & 9.0 & 8.4 \\
\hline & & 250 & 7.5 & 5.4 & 4.1 & 8.9 & 8.7 & 8.2 \\
\hline & & 300 & 7.4 & 5.3 & 4.2 & 8.7 & 8.3 & 7.9 \\
\hline \multirow{9}{*}{25} & \multirow{9}{*}{$\begin{array}{l}\text { FBP Filter } \\
\text { Cutoff }\end{array}$} & 0.1 & 9.3 & 9.4 & 9.2 & 21.3 & 31.6 & 31.9 \\
\hline & & 0.2 & 4.8 & 4.2 & 3.9 & 24.3 & 22.0 & 20.5 \\
\hline & & 0.2 & 4.5 & 3.5 & 3.0 & 21.6 & 19.3 & 17.6 \\
\hline & & 0.3 & 4.3 & 3.2 & 2.7 & 20.3 & 16.7 & 14.2 \\
\hline & & 0.4 & 4.5 & 3.5 & 3.1 & 20.2 & 16.3 & 13.4 \\
\hline & & 0.5 & 4.7 & 3.8 & 3.4 & 20.2 & 16.2 & 13.3 \\
\hline & & 0.6 & 4.8 & 4.0 & 3.5 & 20.1 & 16.2 & 13.3 \\
\hline & & 0.8 & 5.2 & 3.9 & 3.8 & 19.9 & 16.1 & 13.3 \\
\hline & & 1 & 4.5 & 4.1 & 4.0 & 20.2 & 16.1 & 13.3 \\
\hline \multirow{2}{*}{30} & \# updates & 150 & 8.7 & 6.0 & 4.3 & 10.4 & 9.8 & 8.4 \\
\hline & FBP cutoff & 0.4 & 5.4 & 4.1 & 3.2 & 23.5 & 18.4 & 14.5 \\
\hline
\end{tabular}

Note: The filter type used for FBP was Butterworth with order 5. The FWHM of the Gaussian post-smooth in OSEM3D was set to 2 times the pixel size, respectively.

Note that with using about 20 OSEM updates the FWHM is already below the value of convergence in FBP.

Increasing the orbit radius results in worse performance in both uniformity and resolution for LEAP and LEHR 
collimation, whereas the LEUHR collimator shows lower sensitivity with respect to the radius and with Flash3D reconstruction the image quality measures are rather unaffected by the distance of the detector to the object.

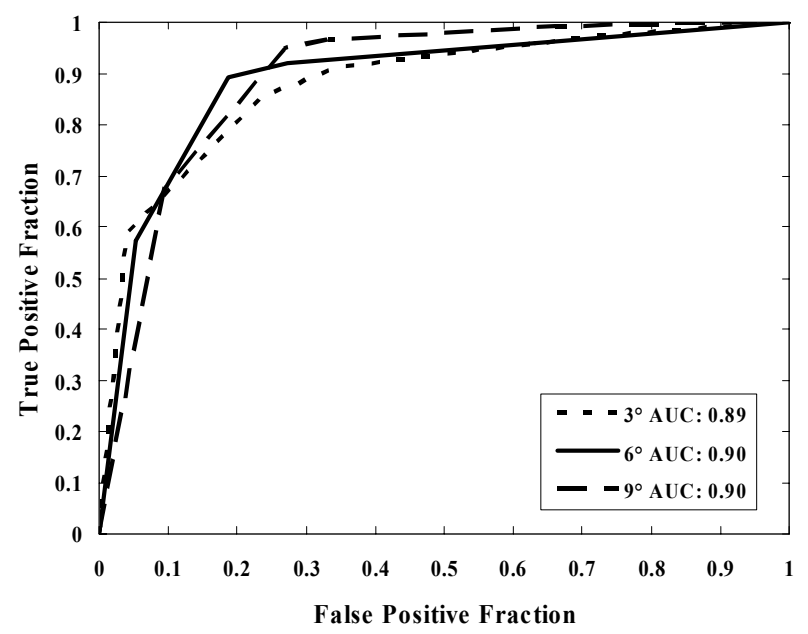

Fig.4. ROC curves (average points of five readers) for three different angular steps (36 OSEM updates).

In Fig. 4 the ROC curves for three different angular steps are shown using the data sets of the torso reconstructed with 36 updates (see Table 3). Note that, even though the area under the curve stays in the same range, the ROC curves intersect which may indicate slightly different performance in terms of specificity and sensitivity and will be discussed later.

Fig. 5 to 7 show results of the ROC studies compared with quantitative measures which were obtained by an analysis of the normal cases read in the observer studies. In Fig.5 the area under the ROC curve (AUC) is given for three different angular steps and three OSEM iteration levels. Fig. 6 shows the standard error (calculated using bootstrap) of these AUC values which give the detection variability and can be seen as an indicator for reader confidence. These ROC characteristic values are plotted against the myocardial nonuniformity, respectively, in order to reveal potential correlations. The $\mathrm{x}$ error bars in both graphs give the standard error of the nonuniformity and the y-error bars show the standard error of the AUC (Fig.5) and its standard error (Fig.6).

Fig.7 combines two quantitative measures, myocardial nonuniformity and wall FWHM, to compare it with the lesion detection performance. The values for 3 degrees angular step are shown for the three investigated iteration levels. The left ordinate shows nonuniformity and AUC in the same scale and the right ordinate the myocardial wall FWHM.

The resolution recovery improves with increasing number of updates, yet uniformity worsens. The AUC value remains rather unaffected in areas with lower resolution and nonuniformity. A collective result derived from all three graphs (Fig.5 to 7) is a decrease in detection ability and reader confidence above a nonuniformity level of approximately $11 \%$.

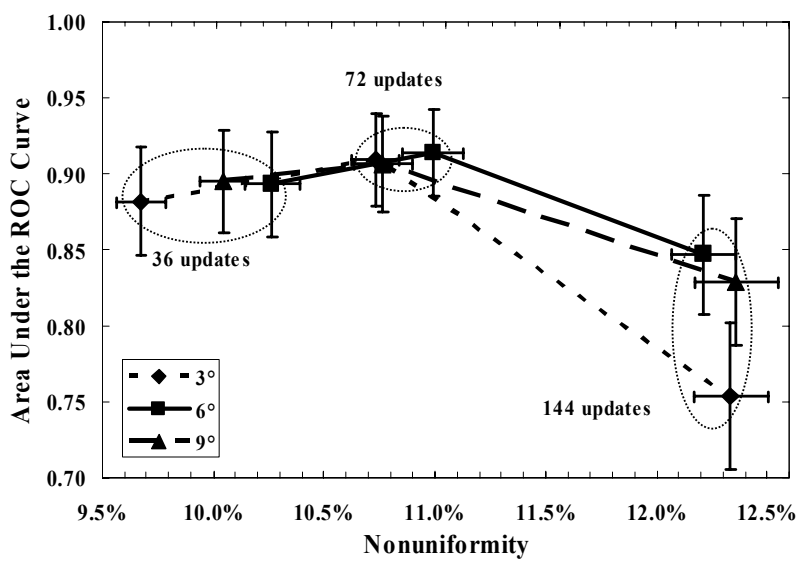

Fig.5. The area under the ROC curve (AUC) for three different angular steps and three iteration levels versus the average non-uniformity in the myocardium of the normal cases.

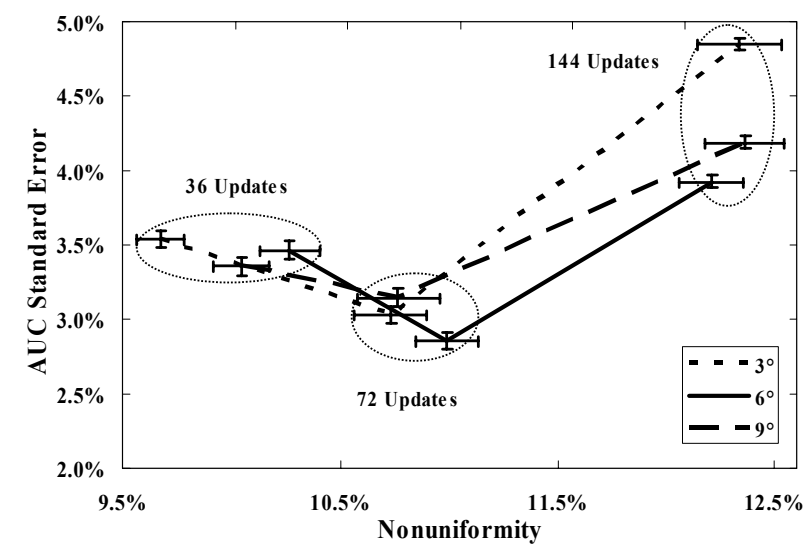

Fig.6. Correlation of the standard error of the area under the ROC curve (AUC) and the non-uniformity for three different angular steps and number of updates, respectively.

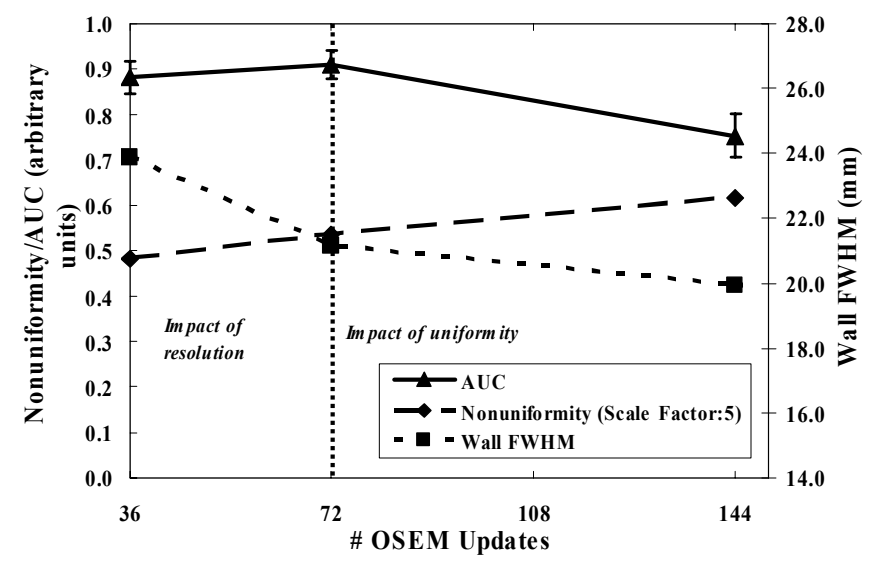

Fig.7. Correlation of myocardial uniformity and resolution and the detection ability ( $3^{\circ}$-Sampling).

\section{DISCUSSION}

The methods used for image data creation are based on real acquired data and thus avoid uncertainties in system modeling 
occurring in simulations. Nevertheless, limitations may occur with respect to parameters that can be varied due to the fact that these variations are constrained to the initially acquired datasets. However, using this data for generating multiple count reduced derivatives by angular extraction and binomial subsampling seems to be an adequate and simple approach for creating a population of data sets. In addition, the processing tool dedicated for real data sets in DICOM-format provides capabilities for use in clinical research applied to patient data in retrospective studies [15].

A statement which can be derived from the quantitative analysis is that the Low Energy Ultra High Resolution collimator shows in general higher stability in terms of resolution and uniformity. However, due to its lower sensitivity it might not be suitable for tasks where very low count rates are expected.

Concerning reconstruction methods the advantage of OSEM3D in terms of resolution recovery clearly can be seen, however the reconstruction results are in general highly dependent on the parameters used (i.e. number of updates). Thus, for optimal reconstruction results obtained with OSEM3D some experience is required and the target application always should be kept in mind.

By comparing the AUC for 36 updates and different angular steps in Fig.5 variations in angular sampling from 3 to 9 degrees have only little impact on the overall detection ability of the defect. However, considering the intersection of the corresponding ROC curves in Fig.4 the AUC value per se does not always give full insight in terms of detection performance. For these cases proposals for extensions of the ROC method exist [13]. For example, if one is interested in high specificity or sensitivity decisions partial areas under the ROC curve could be considered. In our case given a desired sensitivity above 0.8 (corresponds to the partial AUC for TPF over 0.8) the 3 degree sampling would result in a worse performance than 6 and 9 degrees. On the other hand, with a desired specificity of over 0.8 (corresponds to the partial AUC for a FPF below 0.2) 3 and 6 degree sampling performs slightly better.

Fig.5-7 show that there are correlations between quantitative measures and the detection ability. Both myocardial wall resolution and uniformity affect the detection performance, whereas the myocardial uniformity seems to be more dominant. It appears that beyond a nonuniformity threshold of approximately $11 \%$ the detection ability and reader confidence are highly dependent on uniformity. Thus, in this area a more precise prediction of ROC behavior based only on available quantitative results may be possible.

Altogether, we see correlations between quantitative measures and the detection ability for this particular lesion setup. However, in order to make a more general statement, defects have to be varied in size contrast and position. In addition, the AUC values used in these studies in general were rather high, although the smallest available lesion, provided by Data Spectrum, and low contrast and count levels were used.
More clinical AUC values $(\approx 0.75-0.85)$ can be achieved with patient populations and unknown lesion positions [Tsui].

\section{CONCLUSION}

Our data processing methods and quantitative analysis show a meaningful and efficient approach to characterize image affecting parameters based on real data. The newly developed quantitative cardiac analysis tool [14] demonstrates its practicability and shows proper sensitivity to reveal relevant image quality aspects.

In terms of correlating visual detection ability with quantitative results some potentials were shown. We found that the outcome of human ROC studies cannot be predicted absolutely with only quantitative measures available, since observer studies are influenced by too many factors whereas the main variability is caused by the human himself. However, with measures like resolution and uniformity, relative differences in AUC values can be anticipated. Results show that in general myocardial uniformity has a stronger effect on the detection ability than wall resolution.

\section{ACKNOWLEDGMENT}

The authors would like to thank the participants in the observer study, Günter Hahn for valuable discussion and the Systems Test team at Hoffman Estates for their help during the acquisition of the data.

\section{REFERENCES}

[1] B. Hesse et al, "EANM/ESC procedural guidelines for myocardial perfusion imaging in nuclear cardiology",EJNM, 32(7), 2005, p.855

[2] FJ Kocke et al.," ACC/AHA/ASNC Guidelines for the Clinical Use of Cardiac Radionuclide Imaging”, ACC/AHA/ASNC Practice Guidelines, 2003

[3] MK O'Connor, CB Hruska, "Effect of tomographic orbit and type of rotation on apparent myocardial activity.",NMC 26(1), 2005, p.25

[4] C Kamphuis, FJ Beekman, MA Viergever, "Evaluation of OS-EM vs. ML-EM for 1D, 2D, and fully 3D SPECT reconstruction", IEEE TNS, 44(3), 1996, p.2018

[5] Y Takahasji, et al.,"Evaluation of the number of SPECT projections in the ordered subsets-expectations maximization image reconstruction, Ann. Nuc. Med. 17(7), 2003, p.525"

[6] JA Bieszk, EG Hawman, "Evaluation of SPECT Angular Sampling Effects: Continuous Versus Step-and-Shoot Acquisition”, JNM 28, 1987, p. 1308

[7] MW Groch, WD Erwin, "SPECT in the Year 2000: Basic Principles." Journal of Nuclear Medicine Technology Volume 28(1), 2000, P233244

[8] J.A. Hanley, B.J. McNeil, "The Meaning and Use of the Area under a Receiver Operating Characteristic (ROC) Curve", Radiology 143: 29-36, April 1982

[9] S. Sankaran, E.C. Frey, et al, "Optimum Compensation Method and Filter Cutoff Frequency in Myocardial SPECT: A Human Observer Study", JNM 43, 2002, p.432

[10] K.L. Gilland, B.M.W Tsui, et al, "Comparison of Channelized Hotelling and Human Observers in Determining Optimum OS-EM Reconstruction Parameters for Myocardial SPECT”, IEEE TNS, 53(3), 2006, p.1200

[11] M.V. Narayanan, M.A. King, et al, "Human Observer ReceiverOperating-Characteristic Evaluation of Attenuation, Scatter, and Resolution Compensation Strategies for ${ }^{99 \mathrm{~m}} \mathrm{Tc}$ Myocardial Perfusion Imaging”, JNM 44, 2003, p.1725 
[12] E.C. Frey, K.L. Gilland, et al., "Application of task-based measures of image quality to optimization and evaluation of three-dimensional reconstruction-based compensation methods in myocardial perfusion SPECT", IEEE Transactions in Medical Imaging 2002; 21(9):1040-1050

[13] B. D. Gallas, "Reader Studies", 2006 IEEE MIC-NSS, www.gamma.radiology.arizona.edu/IQ/page8/assets/Observers.pdf

[14] JT Chapman, AH Vija, EG Hawman, J. Zeintl, J. Hornegger, "A Quantitative Method for Assessing Performance of Cardiac Imaging Systems ",Submission to 2006 IEEE MIC

[15] AH Vija, JT Chapman, J. Zeintl, EG Hawman," Development of Rapid SPECT Acquisition Protocol for Myocardial Perfusion Imaging", Submission to 2006 IEEE MIC 